\title{
Reduced Structural Modeling of Integrally-Strained Slender Wings
}

\author{
Rafael Palacios* and Carlos E.S. Cesnik ${ }^{\dagger}$ \\ Department of Aerospace Engineering, University of Michigan. Ann Arbor, Michigan, USA
}

\begin{abstract}
This paper introduces a procedure to model the dynamics of active slender structures with embedded actuators and sensors. The objective is to construct a loworder high-accuracy formulation for easy parameterization of the design space in nonlinear aeroelastic analysis. Main assumptions are small local strains and local rotations, large deflections and global rotations, quasielectrostatic behavior, linear electroelastic constitutive relations, and slenderness of the structure. The model also includes anisotropic material properties, electroelastic coupling, hygrothermal effects, and is integrated with the vehicle flight dynamics. The condition of slenderness is used to define a three-step analysis scheme based on asymptotic approximations to the electroelastic field, which can adequately represent the passive or active structural dynamics of a quite general class of air vehicles, including future morphing vehicle concepts. Two main configurations are investigated in this work: a rotor blade with embedded piezoceramics (Active Twist Rotor), and a joined-wing configuration for unmanned SensorCraft. On the Active Twist Rotor, a deformation mode for the camber bending is added to classical beam strain measures. Then, the response of this typical plate mode to the embedded actuation is studied and quantified within a 1-D model. On the SensorCraft, the structural characterization of the aircraft with joinedwing and flexible fuselage are investigated and compared to detailed 3-D build-up finite-element models.
\end{abstract}

\section{Introduction}

Conventional wisdom in the design of complex physical systems calls for an orderly simplification of the mathematical models used to describe them. Determination of the appropriate degree of this simplification comes from a tradeoff between the stage

\footnotetext{
*Graduate Student, F.-X. Bagnaud Fellow (rpalacio@umich.edu)

${ }^{\dagger}$ Associate Professor (cesnik@umich.edu). Associate Fellow, AIAA

Copyright $\odot 2004$ by Rafael Palacios and Carlos E. S. Cesnik. Published by the American Institute of Aeronautics and Astronautics, Inc., with permission.
}

within the design process and the inherent complexity of the system, and the efficiency of this reduction becomes critical in terms of aggregated time and monetary costs to the complete design cycle. The aeroservoelastic analysis of aircraft is a paradigmatic case of the importance of these efficient reduced-order models, and a large amount of research is devoted to the generation of the optimumsize models for the control, structural and aerodynamic subsystems.

This work presents a systematic process of generation of reduced models for aeroelastic analysis of active structures with embedded actuators and sensors at the conceptual stage in the design process. Thus, it allows an easy parameterization of the main geometrical (type of airfoil, location and connection of major elements - body and lifting surfaces), and material characteristics (lay-ups of composite laminates, location and type of embedded actuators) of the aircraft subcomponents, upon the assumption of overall slenderness of each of them. This is the case in a quite general class of air vehicles, which includes high-altitude long-endurance (HALE) aircraft, helicopter rotors, and large transport aircraft. In the reduced model, the characteristics of each structural component are reduced into the evolution of a set of variables along the reference line. This analysis is performed with a broader characterization than in typical beam models, since local 3-D information is carried in the process to compute the electroelastic fields on the original structure. This also allows an arbitrary definition of the variables of interest in the 1-D problem, what can be especially effective in the design and analysis of variable geometry structures like the ones found in proposed morphing concepts ${ }^{1}$.

Basic concepts in structural beam theory were first developed for the modeling of homogeneous isotropic structures. Classical formulations such as Euler-Bernoulli or Timoshenko theories are based on specific assumptions on the displacement field that represents the behavior of those particular cases. These theories are examples of displacement formulations: an ad hoc approximation of the cross-sectional displacement field yields the strain energy, and using energy principles certain beam stiffness relations can be defined, as well as the subsequent equations of motion. Classical assumptions in the displacement field, however, are poor 
representations of the behavior of composite beams. The concept of warping, first developed for the Saint-Venant theory of torsion, is used then to quantify this mismatch ${ }^{2}$, and based on it, a number of composite beam theories have been formulated ${ }^{3,4}$. The formal way to tackle the mathematical problem of the dimensional reduction of a 3-D field without ad hoc assumptions is the use of asymptotical expansions in the small parameters of the system. Its application in slender elastic solids is the Variational-Asymptotic Beam Sectional analysis $\operatorname{method}^{5,6}$, which has been successfully used for the modeling of composite beams. Using this formulation, the warping field is obtained as part of the solution process, which gives a superior characterization of the actual deformation process compared to other formulations of the problem.

Beam models are also desirable in the case of active slender structures with distributed strain actuators (viz., active beams). However, distributed actuation will easily yield non-classical modes of deformation in the structure $^{7,8}$. These will influence, for instance, the aeroelastic response of the system through their couplings with the aerodynamic field. Including these effects in a 1$\mathrm{D}$ model is a rather complex task, and active structures are conventionally analyzed using plate models, even when their slenderness ratio is high ${ }^{9}$. A 1-D representation of the non-classical deformation of active beams is pursued in the present work, with the ultimate goal of using it in the construction of a reduced-order model for aeroservoelastic analysis and design.

Different approaches to the modeling of non-classical effects in refined composite beam analysis can be found in the literature. Some formulations ${ }^{8,10}$ present higherorder beam variables as the way to keep the classical assumptions for the isotropic representation of the beam in the classical (low-order) variables. Although these approaches capture the approximate warping field, they yield artificially large systems. It can be mathematically shown ${ }^{11}$ that the classical variables (extension, torsion and bending) provide the best representation of the lowfrequency dynamics of closed-cell anisotropic beams.

A few other models of composite beams, however, indeed look into a physically higher-order problem. In these cases the addition of non-classical variables to the problem brings into evidence some features of the system: camber deformation of thin plates under isotropic actuators $^{12}$, or the Vlasov correction for open cross sections $^{13}$. Previous work $^{14}$ introduced the concept of arbitrary cross-sectional deformation modes, within the context of the variational-asymptotic formulation. The formulation finally defined an eigenvalue problem in the components of the cross-sectional strain energy to compute those modes. The original eigenvalue problem is substituted here by an assumed modal basis (as in the
Rayleigh-Ritz method) for the non-classical deformation mode shapes. Note that, while the classical modes represent the possible deformations of an elastic line in space (zero order in the asymptotic expansion), the nonclassical effects can be associated to the finite size of the cross-section, called here finite-section modes. They will be in principle arbitrary in shape, providing the most general representation of physically higher-order components of the deformation.

\section{Active Beam Formulation with Finite-Section Modes}

In the process of dimensional reduction of slender structures through the variational-asymptotic method, the 3-D electroelastic problem can be approximated by two independent problems ${ }^{5}$, as shown in Figure 1: a 2-D analysis at each cross section (curvilinear coordinates $x_{2}$ and $x_{3}$ ) and a 1-D analysis along the reference line (coordinate $x_{1}$ ). The final 3-D elastic and electric fields are recovered by combination of the results of these two subproblems. Previous works by the authors have set up the characteristics of both the $2-\mathrm{D}^{7}$ and the $1-\mathrm{D}^{15}$ problems for active slender structures. A brief review is included here from a global perspective.

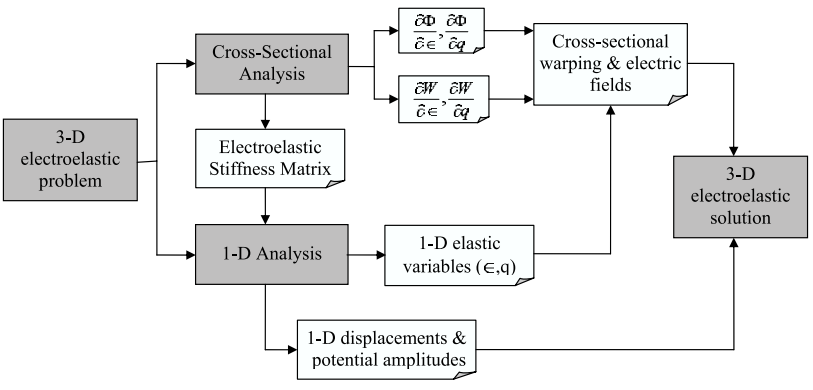

Figure 1. Three-step solution to the 3-D electroelastic problem

\section{Cross-sectional analysis}

First, the reference line is chosen in the undeformed structure along the dominant dimension. Its definition is in principle arbitrary, and a plane cross section can be associated to each point $x_{l}$. The cross sections do not need to be normal to the reference line (for instance, they can follow the aerodynamic airfoils in a swept wing).

According to the variational-asymptotic method $^{5}$, the constitutive relations in the motion of the reference line can be now obtained by the minimization of the electric enthalpy per unit arc length. Under the assumption of applied electric fields in the direction of polarization of the piezoelectrics, the cross-sectional electric enthalpy is given by the functional ${ }^{16}$

$$
H=\frac{1}{2} \int_{C S}(\Sigma: \Gamma-D \cdot E) \cdot d x_{2} d x_{3}
$$

Page 2 
where $\Gamma$ is the local strain tensor, $E$ is the local electric field. $\Sigma$ and $D$ are their force conjugates (stress and electric displacements). The domain of integration is the local cross section at $x_{1}$. It is assumed that the local electroelastic problem is linear (small strains and local rotations, and linearized electric fields), although geometrical nonlinearities may appear in the global 1-D problem.

The 1-D representation of the deformation of the reference line is chosen at this point. The baseline are naturally the intrinsic deformation magnitudes for the elastic curve (extension along $x_{1}$, torsion about $x_{1}$, and bending in $x_{2}$ and $x_{3}$ ), given in the form of a vector of classical strain measures, $\in\left(x_{1}\right)=\left\{\gamma_{11}, \kappa_{1}, \kappa_{2}, \kappa_{3}\right\}$. In addition to them, a set of finite-section deformation modes are defined using assumed distributions of displacements in the cross section, $\psi_{q}\left(x_{2}, x_{3}\right)$. The vector of their amplitudes, $q\left(x_{1}\right)$, adds elastic variables to the final 1-D model. In a similar way, the electric actuation is defined by a set of electric modes in the cross-sectional distribution of electric potential $\psi_{v}\left(x_{2}, x_{3}\right)$, being the corresponding amplitudes $v\left(x_{1}\right)$. The local strain tensor and electric field of equation (1) can then be expressed by the following exact (linear) relations:

$$
\begin{aligned}
& \Gamma=\Gamma_{\in} \in+\Gamma_{w}\left(\bar{w}+\psi_{q} q\right)+\Gamma_{w^{\prime}}\left(\bar{w}^{\prime}+\psi_{q} q^{\prime}\right) \\
& E=-\nabla\left(\bar{\phi}+\psi_{v} v\right)
\end{aligned}
$$

where the $\Gamma$-operators are linear operators and are defined in Ref. 6. Equation (2) connects the electroelastic description of the 3-D solid $\{\Gamma, E\}$ with the set of 1-D variables in the reduced structure $\left\{\in, q, q^{\prime}, v\right\}$, through the inclusion of unknown warping and induced electric potential fields $\{\bar{w}, \bar{\phi}\}$ throughout the current cross section. Both fields are discretized using a finite-element representation in the cross section and are finally solved as a function of the 1-D variables by an asymptotic minimization of the electric enthalpy. The small parameter in the asymptotic approximation is the inverse of the slenderness ratio, $\mathrm{SR}^{-1}=\mathrm{h} / \mathrm{L}$ ( $\mathrm{h}$, cross-sectional typical dimension; L, typical dimension along the reference line). The results of this linear optimal problem are, first, the warping and electric influence coefficients (WIC and EIC, respectively), defined as the local 3-D displacement field at the cross section related to each 1-D variable, or

$$
\begin{gathered}
W I C=\frac{\partial \bar{w}}{\partial \lambda} \quad \text { and } \quad E I C=\frac{\partial \bar{\phi}}{\partial \lambda} \\
\text { with, } \lambda^{T}=\left\{\begin{array}{llll}
\in^{T} & q^{T} & q^{\prime T} & v^{T}
\end{array}\right\}
\end{gathered}
$$

Second, using these influence coefficients, the enthalpy (1) can be written as a bilinear operator in the 1-D variables, what defines a generalized electroelastic stiffness matrix, $S$, as ${ }^{7}$

$$
H=\frac{1}{2} \lambda^{T} \cdot S \cdot \lambda+O\left(\frac{h}{L}\right)^{2}
$$

The mass matrix corresponding to the selected 1-D description of the beam kinematics is obtained by a similar procedure, starting with the kinetic energy per unit length ${ }^{7}$.

\section{Geometrically-nonlinear dynamics of the reference line}

The dynamics of the resulting 1-D electroelastic system are analyzed under the following hypotheses $\left.{ }^{15}: 1\right)$ large displacements and global rotations of the reference line, 2) small values of strains and local rotations; 3) small amplitudes of the finite-section modes, q;4) prescribed electric potential at the electrodes.

The kinematics of the reference line is determined by the geometrically-exact nonlinear description of Danielson and Hodges ${ }^{17}$, with a representation of large rotations using Rodrigues parameters, $\theta\left(t, x_{l}\right)$. Differentiation in time of the generalized displacement variables $\left\{u\left(t, x_{1}\right), \theta\left(t, x_{1}\right)\right\}$ yields the linear and angular velocities, $\left\{V\left(t, x_{1}\right), \Omega\left(t, x_{1}\right)\right\}$. The amplitudes of the finitesection modes, $q\left(t, x_{l}\right)$, are then superimposed, as a general way to add higher-order deformations. This results in a 1-D description of the dynamics based on the variables of Table 1.

\begin{tabular}{lr}
\multicolumn{2}{c}{ Table 1. 1-D sets of variables } \\
\hline Displacements & $\{u, \theta, q\}$ \\
Velocities & $\{V, \Omega, \dot{q}\}$ \\
Strains & $\left\{\gamma, \kappa, q^{\prime}\right\}$ \\
\hline
\end{tabular}

This set of variables is used to construct the kinetic energy per unit length, $T$, and the work per unit length of the external applied forces, $W$, which, together with the electric enthalpy $H$ of equation (4), determine the dynamics of the reference line. Hamilton's variational principle for electromechanic systems is used for this purpose. For each 1-D continuous subcomponent of the structure, it can be expressed as follows

$$
\int_{t_{1}}^{t_{2}} \int_{0}^{l}[\delta(T-H)+\overline{\delta W}] d x_{1} d t=\overline{\delta A}
$$

where $t_{l}$ and $t_{2}$ are fixed times, $l$ is the arc length of the reference line. $\overline{\delta A}$ is the virtual action at the ends of the beam and at the ends of the time interval, and $\delta$ is the variational operator. Bars are used over magnitudes that do not correspond to the variation of a function. 
The weak form of this variational description is used to construct a spatial finite-element discretization along the reference line on the variables of Table 1 (the actual implementation in Ref. 15 used the force and momentum conjugates of strains and velocities). In symbolic form, and for the case of non-follower loads, the resulting timedomain nonlinear equations can be expressed as

$$
A(X) \cdot \dot{X}=F_{S}\left(X, \hat{X}_{x=0}, \hat{X}_{x=l}\right)-F_{L}
$$

Here, $A$ is the inertia matrix operator, $F_{s}$ is the structural vector operator, and $F_{L}$ is the force vector operator. $X$ is the state vector of the values of the 1-D variables at each element, being $\hat{X}$ its boundary values. The variational formulation also yields the natural boundary conditions in the system:

$$
B C\left(\hat{X}_{x=0}\right)=0 \text { and } B C\left(\hat{X}_{x=l}\right)=0
$$

Given the external loading, the excitation to the embedded actuators and the boundary conditions (7), equation (6) describes the nonlinear dynamics of a single beam. In multi-beam configurations, some of the boundary conditions are substituted by relations between the boundary values of the different beams at each joint. Note that the boundary and joint conditions are imposed in displacement, rotations, and also in the finite-section amplitudes, as well as in their corresponding strains (or force conjugates).

\section{Recovery of the 3-D electroelastic solution}

In the last stage of this three-step solution process, the previous results are combined at each time step to recover the actual 3-D displacement and electric potential fields in the original active structure. Let $r(t, x)$ be the instant position vector of a point $x=\left(x_{1}, x_{2}, x_{3}\right)$ in the undeformed structure (which can be also moving as a rigid body), and let $\xi=\left(0, x_{2}, x_{3}\right)$ be the position of that point in the undeformed cross section. Its position vector after the deformation is given by:

$$
\begin{aligned}
R(t, x) & =r\left(t, x_{1}\right)+u\left(t, x_{1}\right)+\mathrm{C}\left(\theta\left(t, x_{1}\right)\right) \cdot \xi+ \\
& +\psi_{q}(\xi) \cdot q\left(t, x_{1}\right)+W I C(\xi) \cdot \lambda^{s}\left(t, x_{l}\right)
\end{aligned}
$$

where $\mathrm{C}$ is the rotation matrix corresponding to the vector of Rodrigues parameters, $\theta$, and $\lambda^{s}$ are the structural 1-D variables $\left\{\in, q, q^{\prime}\right\}$. These displacements can be used directly in the definition of the 3-D fluid-structure interface for aeroelastic analysis, eliminating the need for extrapolations typically used by beam models (e.g. Ref. 18). The 3-D electric field is obtained in a similar way.

This process also yields the 3-D stress/strain fields corresponding to the deformation field. To avoid derivation of (8) at each time step, a set of stress/strain influence coefficients analogous to the WIC can be evaluated in the cross-sectional analysis.

\section{Applications}

The cross-sectional formulation has been implemented in the computer code UM/VABS, which, together with an in-house time-domain beam solver, is used in the following numerical studies. Some typical configurations with embedded actuators are used herein to exemplify the proposed configuration. The objective is to show both the accuracy and the flexibility of the present reduced structural model to describe complex deformations of slender structures. Note that the finite-section modes are so far of arbitrary shape and it is at this point where they have to be numerically defined for a given problem as displacement fields of the cross-sectional finite-element mesh.

\section{Camber-bending deformation in 1-D models of a thin composite strip with surface-mounted isotropic actuators}

This simple case illustrates a typical configuration in which a finite-section mode may be used to capture unconventional deformations. Consider a graphite-epoxy thin strip with constant ply angle $(\phi)$ and surfacemounted piezoelectric (PZT) actuators on the tip region, as shown in Figure 2.

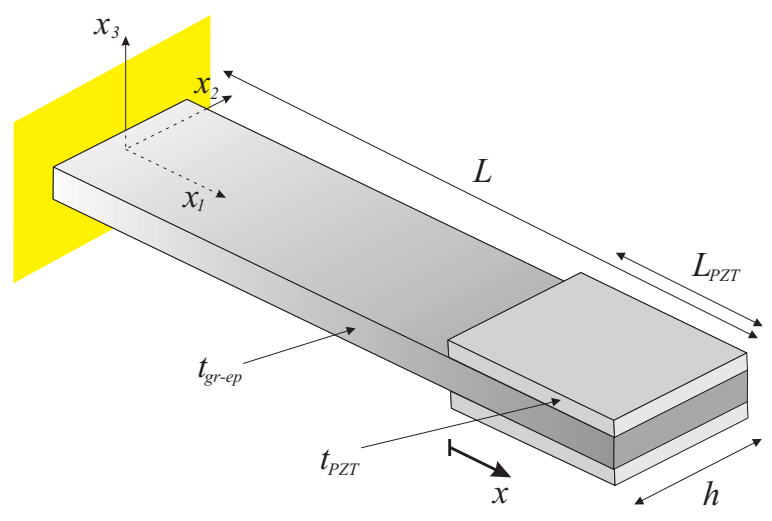

Figure 2. Active thin strip model

This case was experimentally studied by Chandra and Chopra $^{8}$, who showed the relative importance of including the camber bending response in the analysis and subsequently developed a specific formulation to address this non-classical beam problem. In the context of the present formulation, a parabolic finite-section mode can be defined to represent this deformation. The general definition of such mode is

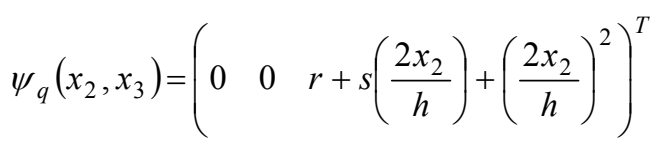

where $x_{2}$ is the chordwise coordinate with reference to mid-chord. The parameters $r$ and $s$ are included to impose orthogonality of the new mode to the classical beam 
deformations (extension, twist and bending) ${ }^{7}$, and they are $r=-1 / 3, s=0$ for the configuration of Figure 2 .

Table 2. Geometry definition of the active thin strip

\begin{tabular}{lrlr}
\hline$h$ & $25.4 \mathrm{~mm}$ & $L_{P Z T}$ & $2 h$ \\
$t_{g r-e p}$ & $2.79 \mathrm{~mm}$ & $L$ & $40 h$ \\
$t_{P Z T}$ & $0.254 \mathrm{~mm}$ & & \\
\hline
\end{tabular}

The geometrical definition given in Table 2 is used in this example. For a ply angle $\phi \neq 0$, the strip shows bending/twist/camber-bending elastic coupling. In a previous work $^{15}$, an analytical solution was found for this problem using a boundary field approach. Here, numerical analyses are carried out using the present reduced active 1-D formulation with a finite-section mode for the camber bending, and an MSC.Nastran plateelement solution with equivalent thermal stresses.
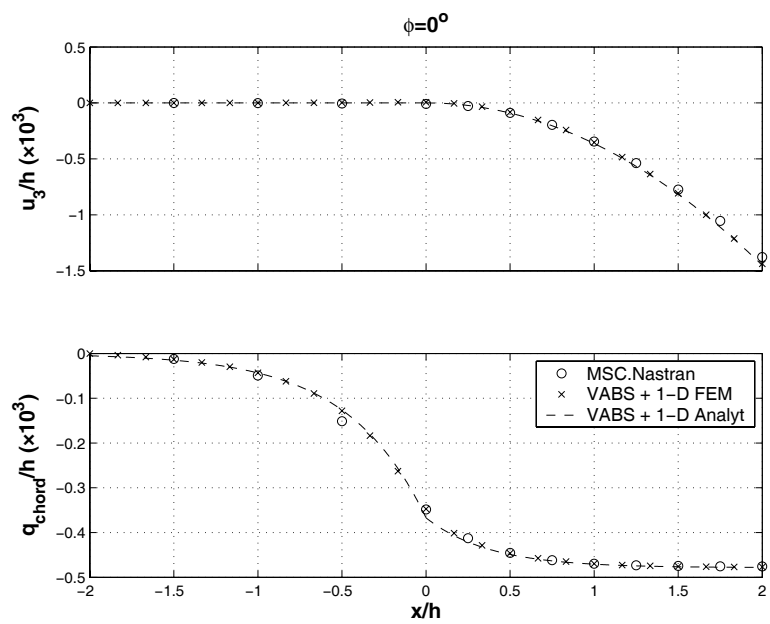

Figure 3. Static response of thin strip with PZT actuators $\left(\phi=0^{\circ}\right)$
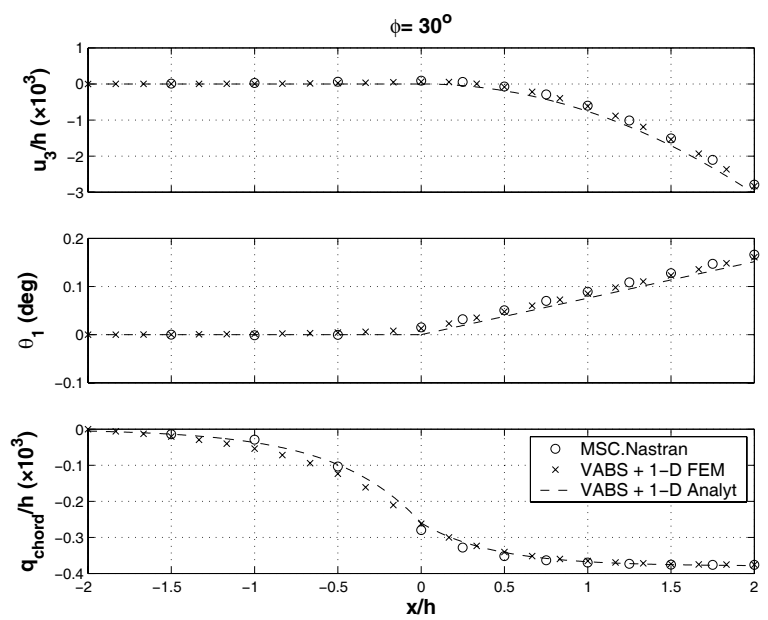

Figure 4. Static response of strip with PZT actuators $\left(\phi=30^{\circ}\right)$

The static response in the 1-D variables of the reduced model (vertical displacement $u_{3}$, twist angle $\theta_{3}$, and amplitude of the camber bending deformation $\mathrm{q}_{\text {chord }}$ ) to a PZT free strain of $240 \mu \varepsilon$ is shown in Figure 3 for plies parallel to the strip longitudinal axis and in Figure 4 for plies at $30^{\circ}$ with this axis. The correlation between the present method ("VABS + 1-D FEM"), the analytical solution of Ref. 15 ("VABS + 1-D Analyt"), and the plate finite-element model ("MSC.Nastran") is excellent, and it demonstrates the capability of the finite-section modes technique to capture non-classical deformations within a low-order formulation.

\section{Camber-bending deformation in 1-D models of active rotor blades}

Integral actuation in flexible slender wings may also induce non-classical deformations. As an illustration, the elastic response including camber-bending to embedded actuation in a composite rotor blade is studied here using the finite-section mode defined in (9).

A configuration based on the Active Twist Rotor (ATR) prototype blade ${ }^{19}$ has been chosen for the analysis. The structural characteristics of the blade cross section are shown in Figure 5, and they are kept constant along its spanwise direction. The composite construction is built on E-Glass and S-Glass around a foam core of Rohacell 711, and includes Active Fiber Composites ${ }^{20}$ (AFC) as embedded anisotropic piezoactuators.

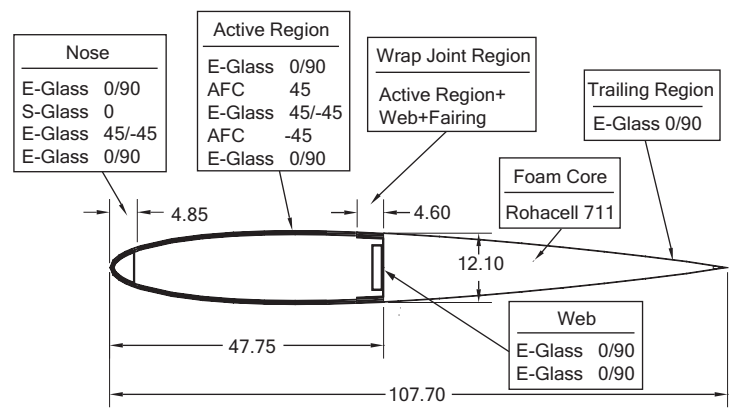

Figure 5. Layup of the ATR model blade (dimensions in mm)

A detailed finite-element model of the cross section was built for UM/VABS. The finite-section mode of (9) was then numerically defined at the nodes of the model, generating the mode shape of Figure 6. Also, for the coming analysis, the individual active plies of Figure 5 are further divided in two regions of independent actuation, as in Figure 7.

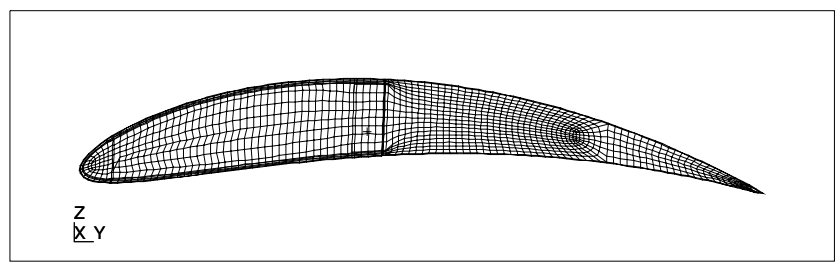

Figure 6. Finite-section mode for camber-bending deformation

Page 5 


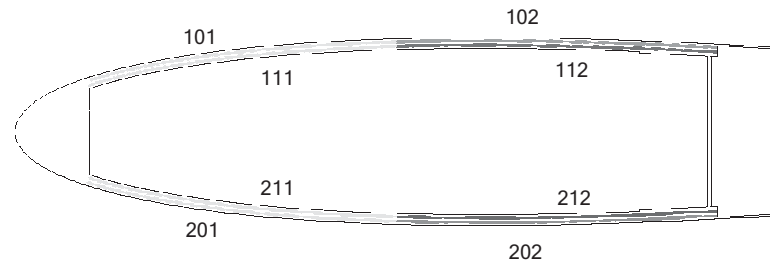

Figure 7. Regions of independent actuation in the ATR model

In order to set the optimum actuation scheme for camber-bending, the active plies are actuated one at a time with a given voltage. Then, the amplitude $q$ of the camber-bending deformation induced by each active ply was computed for the interior beam solution, and is shown in Figure 8. Several conclusions can be extracted from the results: 1) camber deformation is induced with opposite signs of actuation in the upper and lower walls (the same actuation scheme that induces maximum beam bending); 2) rear actuators $(* * 2)$ are more effective for this deformation; 3 ) for this configuration of actuators, the maximum amplitude in camber-bending deformation is about $1 \%$ of the typical response in beam bending.

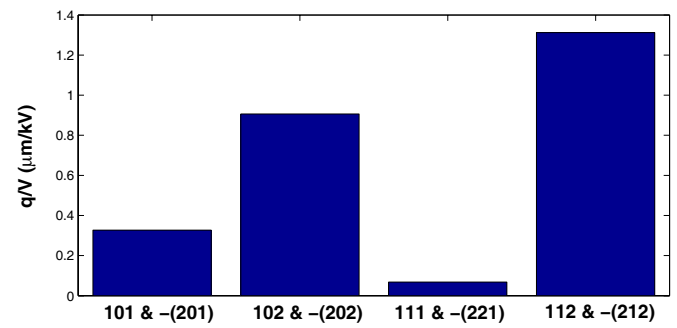

Figure 8. Camber-bending deformation amplitude induced by each region of actuation

Finally, the 1-D static response to the cross-sectional actuation scheme for maximum camber-bending response is analyzed in this blade under hingeless conditions. Only certain active plies along the direction of the beam axis are actuated, as shown in Figure 9 (this differs from the actual ATR prototype blade, where all active plies along the span were connected to the same electric signal). The chosen actuation is $4000 \mathrm{~V}_{\mathrm{pp}}$, with $1.143 \mathrm{~mm}$ of electrode spacing. The ratio of electric excitation between the plies at the upper $(1 * *)$ and lower $(2 * *)$ skins is $2800 \mathrm{~V}$ / $-1200 \mathrm{~V}$.

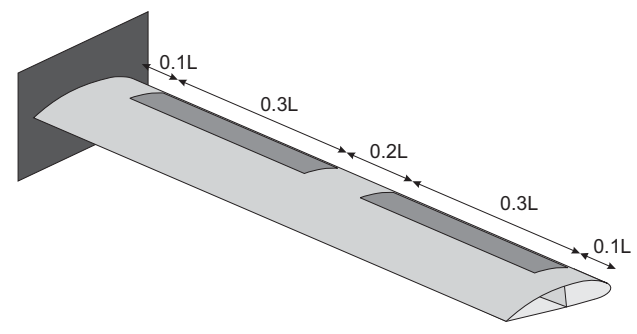

Figure 9. Regions of actuation on the modified ATR blade
Figure 10 shows the 1-D response of the reduced models corresponding to the classical beam analysis and to an extended representation with an additional finitesection mode for camber-bending. In those results, $\mathrm{h}$ is the blade chord $(107.70 \mathrm{~mm})$ and $\mathrm{L}$ is the beam length (1 $\mathrm{m})$. Note that the presence of this new mode substantially modifies the twist response of the beam. However, this perturbation occurs locally at the actuation interfaces, and the twist rate outside these interfaces does not change (according to the variational-asymptotic theory, which determines the asymptotically exact solution in the interior of the domain for each magnitude used to represent the deformation). Finally, the magnitude variations induced by the camber bending in the (spanwise) bending response, which is the main deformation for this actuation, are negligible when compared with the other deformations.

Therefore, non-classical deformations due to embedded actuation may be important, not only because of their actual contribution to the final 3-D displacement fields, but also because of their influence in the response of the main strain measures (extension, twist and bending) of the slender structure.
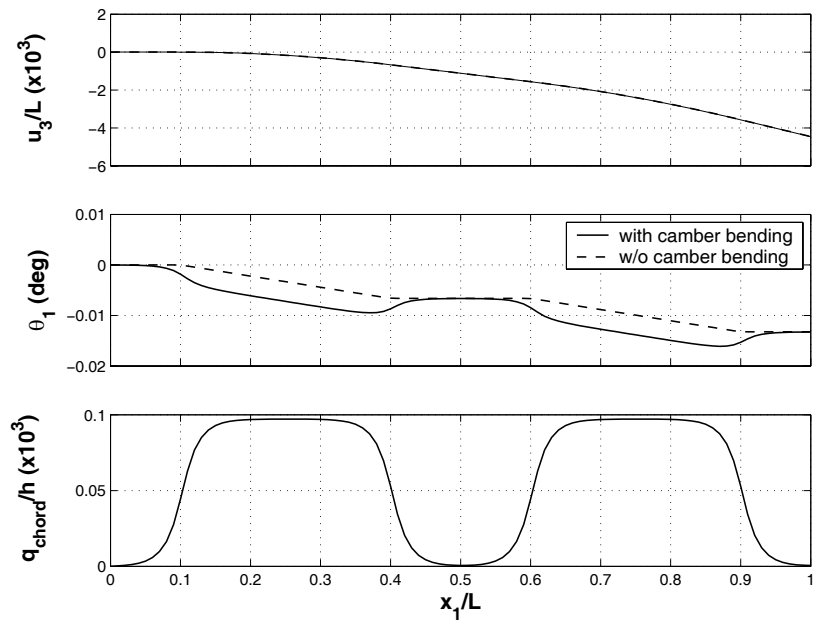

Figure 10. 1-D response to actuation in bending/camber bending (cross-sectional reduction at the midchord point)

\section{Joined-Wing SensorCraft Configuration}

The SensorCraft is an advanced surveillance unmanned air vehicle (UAV) concept currently under investigation by the US Air Force ${ }^{21}$. Among its characteristics, it is desirable that the airframe provides an appropriate geometry $360^{\circ}$ sensor coverage. A possible solution for this requirement is a joined-wing (JW) aircraft configuration, with a geometry that can be defined using the parameterization of Figure 11.

The aeroelastic behavior of this flexible unconventional configuration presents some particular characteristics 
whose possible implications are not yet fully understood. Some fundamental optimization studies have been conducted by Blair and Canfield ${ }^{22}$. These authors have also presented an assessment of the response to some typical loading cases, identifying gust and taxi crater impact as critical ones ${ }^{23}$. In a JW, the aft wing is subject to compressive loads, and its global buckling response and contributions to the aeroelastic instabilities can be critical for the design of the structure. Thus, a geometrically-nonlinear structural model must be consider in the analysis, as it was done by Gallman and $\mathrm{Kroo}^{24}$ for the buckling characteristics, and Cesnik and Brown $^{25}$ for the buckling-aeroelastic interaction. These works have also shown the sensitivity of the buckling onset to the particular design of the interface joint. In addition to this, Livne ${ }^{26}$ has pointed out, among other things, that this configuration may likely present panelflutter instability, and Weisshaar and $\mathrm{Lee}^{27}$ have shown the importance of the interaction of flexible and rigidbody response in flutter analysis on this type of configuration. Summarizing, the aeroelastic analysis of a JW demands a geometrically-nonlinear structural model coupled with the vehicle flight dynamics and an accurate description of the deformation of the wet surfaces to capture local instabilities.

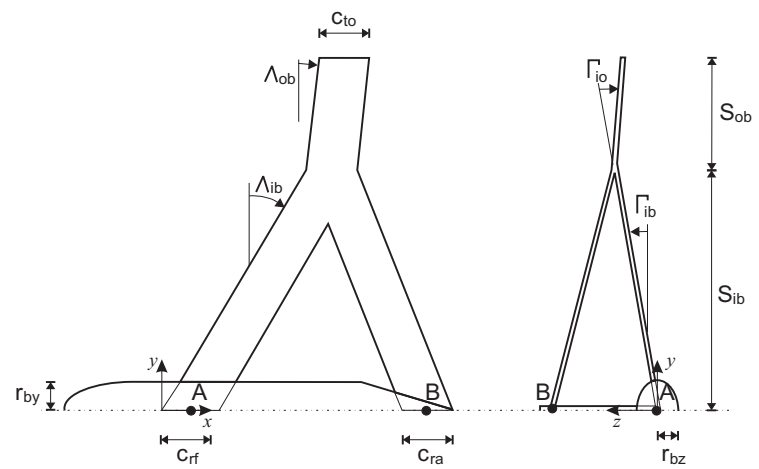

Figure 11. Planform configuration of the Joined-Wing SensorCraft (after Ref. 22)

\begin{tabular}{lrlr}
\multicolumn{4}{c}{ Table 3. Geometric parameters of the JW } \\
SensorCraft configuration \\
\hline $\mathrm{S}_{\mathrm{ib}}$ & $25.40 \mathrm{~m}$ & $\mathrm{C}_{\mathrm{rf}}$ & $2.50 \mathrm{~m}$ \\
$\mathrm{~S}_{\mathrm{ob}}$ & $8.60 \mathrm{~m}$ & $\mathrm{C}_{\mathrm{ra}}$ & $2.50 \mathrm{~m}$ \\
$\Lambda_{\mathrm{ib}}$ & $30^{\circ}$ & $\mathrm{C}_{\mathrm{to}}$ & $2.50 \mathrm{~m}$ \\
$\Lambda_{\mathrm{ob}}$ & $30^{\circ}$ & $\mathrm{X}_{\mathrm{B}}$ & $23.75 \mathrm{~m}$ \\
$\Gamma_{\mathrm{ob}}$ & $9^{\circ}$ & $\mathrm{Z}_{\mathrm{B}}$ & $7.00 \mathrm{~m}$ \\
$\Gamma_{\mathrm{io}}$ & $0^{\circ}$ & $\mathrm{r}_{\mathrm{bz}}$ & $1.00 \mathrm{~m}$ \\
& & $\mathrm{r}_{\mathrm{by}}$ & $1.00 \mathrm{~m}$ \\
\hline
\end{tabular}

In this example, a reduced structural model is developed based on the JW configuration model introduced in Ref. 22. There a parametric finite-element model for MSC.Nastran and MSC.Astros was built using shell elements and then coupled with a panel aerodynamic method. The cross-sectional geometry is based on a LRN1015 airfoil with two spars at $15 \%$ and $85 \%$ of the chord. Spars and skins are made of isotropic material with Young's modulus E=72.4 GPa, Poisson's ratio $\nu=0.3$, and density $\rho=2770 \mathrm{~kg} / \mathrm{m}^{3}$. The material thickness is $2.54 \mathrm{~mm}$, and the geometric parameters that define the planform are included in Table 3.

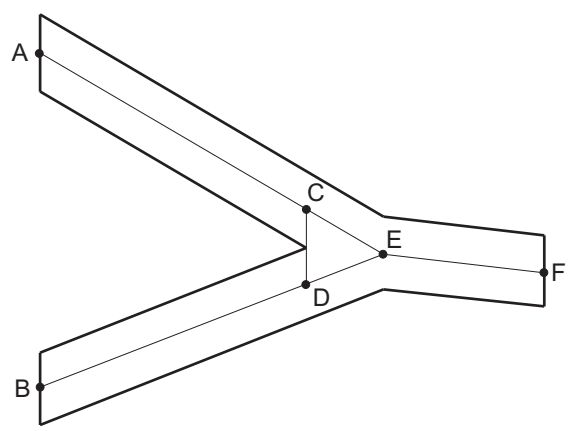

Figure 12. Geometry of the reduced model

The reduced structural model is based on the geometrical representation of Figure 12: segments AC, $\mathrm{BD}$, and $\mathrm{EF}$ are elastic elements, while joint segments $\mathrm{CD}, \mathrm{DE}$, and $\mathrm{EC}$ are rigid connections. The elastic segments are located at the midchord of the wings, and the 1-D variables are the classical beam strains (extension, twist and bending). For each of the three elastic segments, a cross-sectional analysis was first carried out at the corresponding sweep angle $\left(-30^{\circ}, 15^{\circ}\right.$, and $-30^{\circ}$, respectively). That yielded the cross-sectional stiffness and inertia characteristics as well as the warping and stress influence coefficients for each segment. Then the reduced model with these six 1-D subcomponents was solved for clamped conditions at points A and B and different loading cases. Finally the 3-D characteristics of the deformed structure where recovered by combining the 1-D solution and the cross-sectional warping and stress influence coefficients.

Table 4. Static tip displacement and rotations to unit tip loads (x1: outer wing axis; $x 2$ : forward flight direction)

\begin{tabular}{lrrr}
\hline & MSC.Nastran & \multicolumn{1}{c}{$\begin{array}{c}\text { Present } \\
\text { (CDE rigid) }\end{array}$} & \multicolumn{1}{c}{$\begin{array}{c}\text { Present } \\
\text { (joint at E) }\end{array}$} \\
\hline $\mathrm{U}_{3} / \mathrm{F}_{3}$ & $0.216 \mathrm{~m} / \mathrm{kN}$ & $0.231 \mathrm{~m} / \mathrm{kN}$ & $0.251 \mathrm{~m} / \mathrm{kN}$ \\
$\mathrm{U}_{2} / \mathrm{F}_{3}$ & $0.051 \mathrm{~m} / \mathrm{kN}$ & $0.046 \mathrm{~m} / \mathrm{kN}$ & $0.047 \mathrm{~m} / \mathrm{kN}$ \\
$\theta_{1} / \mathrm{M}_{1}$ & $0.0506^{\circ} / \mathrm{kNm}$ & $0.0505^{\circ} / \mathrm{kNm}$ & $0.0602 \% / \mathrm{kNm}$ \\
$\mathrm{U}_{3} / \mathrm{M}_{1}$ & $2.933 \mathrm{~mm} / \mathrm{kNm}$ & $2.804 \mathrm{~mm} / \mathrm{kNm}$ & $2.308 \mathrm{~mm} / \mathrm{kNm}$ \\
\hline
\end{tabular}

The geometry defined in Figure 12 was selected to better represent the characteristics of the shell model of Ref 22. In that work, the 3-D modeling of the joint used a very stiff construction through multiple spars and ribs, which is better captured within the current context using rigid segments as it will be shown next (particularly when compared to the point connection defined in Refs. 25 and 27). Table 4 compares the linear static response to tip unit 
normal force and twist moment for the shell model (MSC.Nastran), the present reduced model with rigid segments (CDE rigid), and a reduced model with a point connection at point $\mathrm{E}$ between the three flexible members (joint at E). As it can be seen, if the rigid-segment joint is used instead of a point joint, the relative error with respect to the shell model solution is reduced from $16 \%$ to $7 \%$ in the tip response to vertical force $\left(\mathrm{u}_{3} / \mathrm{F}_{3}\right)$, and from $19 \%$ to almost zero in the response to twist moment $\left(\theta_{1} / M_{1}\right)$. Therefore, better overall characterization of the joint is achieved by introducing the rigid connections, and this model will be used in what follows.
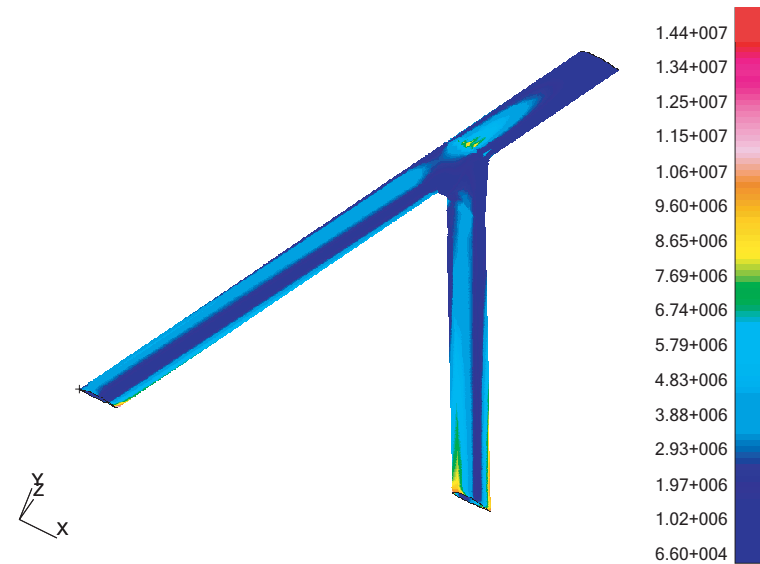

Figure 13. Von Mises stress field (in Pa) for a 1000N normal tip load from the MSC.Nastran solution
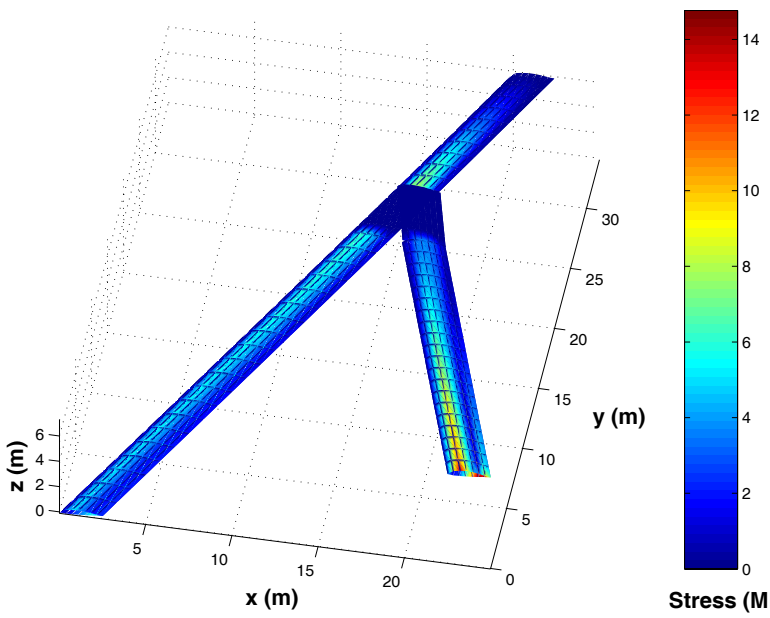

Figure 14. Von Mises stress field for a $1000 \mathrm{~N}$ tip load from the proposed reduced structural formulation

An important feature of the asymptotical reduction scheme is its capability to recover the 3-D electroelastic fields in the original structure. As a first example, the stress fields associated with the linear static response of the JW configuration to a $1000 \mathrm{~N}$ vertical tip load are compared between a shell model in MSC.Nastran (Figure 13) and the present reduced structural formulation
(Figure 14). Agreement is excellent, except for the local effects at the joint, which are beyond the scope of the proposed methodology. For significant 3-D effects like those present at the joint region, a separate detailed FEM model of the region of interest should be created to complement the proposed method.

Next results represent the (factorized) deformed structures in a linear static analysis with MSC.Nastran (Figure 15) and the present reduced model (Figure 16). The loading condition is a constant pressure on the upper skin of the three lifting surfaces (corresponding to segments AC, BD, and EF defined in Figure 12), with relative magnitudes $1,2 / 3$, and $-3 / 2$, respectively. In the reduced structural model, external distributed loads are integrated in the corresponding deformed cross section to yield forces and moments per unit length in the reference line. If finite-section modes were added to the analysis, a corresponding equivalent force per unit length will be obtain from analogous integration.

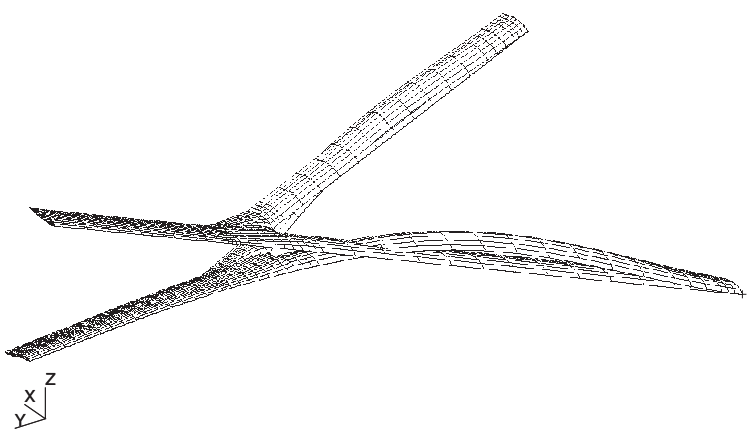

Figure 15. Linear static deformation to pressure loads from the MSC.Nastran solution

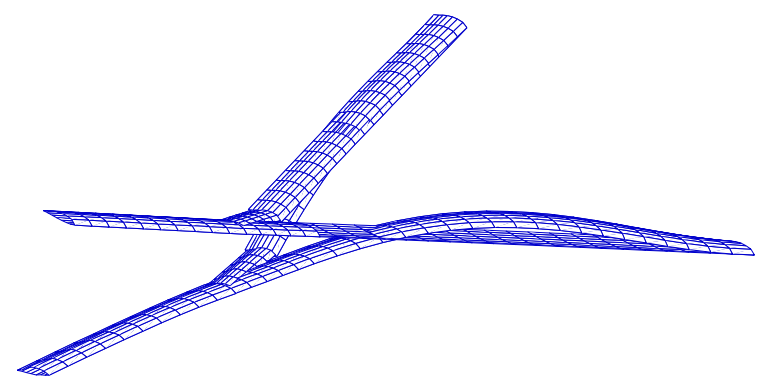

Figure 16. Linear static deformation under pressure loads using the proposed reduced structural formulation

A more quantitative comparison between the 3-D displacement fields obtained by the MSC.Nastran shell model and by the reduced model is shown in Figure 17. It compares the vertical displacement along the leading and trailing edges in the main (front+outer) and the aft wings for unit pressure in the front wing, keeping the ratios $2 / 3$ and $-3 / 2$ for the aft and outer wings, respectively. In this figure, $S$ is the semispan of the main wing. Overall, the proposed reduced modeling technique provides a very 
good estimation of the 3-D displacement field of slender configurations, which is an important requirement for its use in aeroelastic simulations.
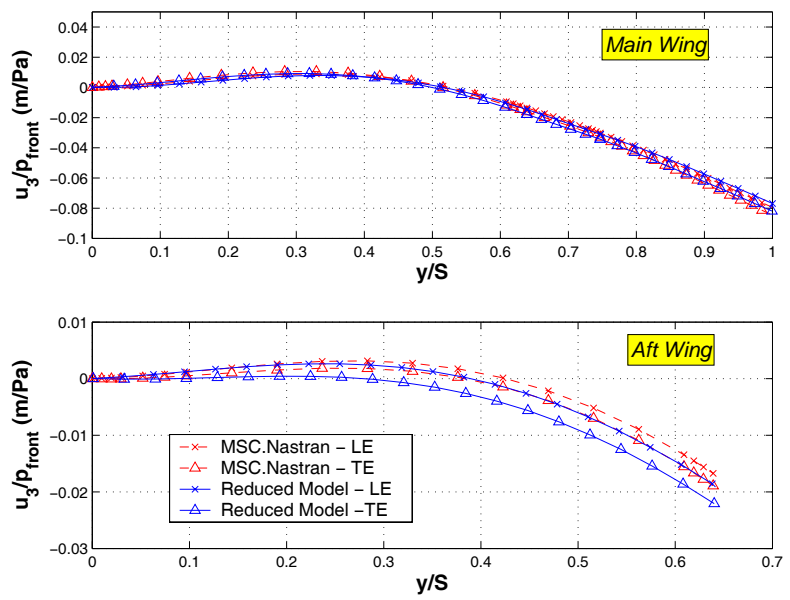

Figure 17. Comparison of vertical displacements in linear static analyses for pressure loads on the leading (LE) and trailing edges (TE) of the joint wings.

The decomposition of the original 3-D elastic problem required about 200 quadrilateral elements for each cross section (this problem is load-independent and needs to be solved only once for a given geometric and material configuration) and 50 1-D elements for the reduced structure. The shell model in MSC.Nastran has about 2200 elements. Although this is not a major issue for the static elastic analysis for a given configuration, there are two major advantages of the present representation: first, parameterization for preliminary design is enormously simplified, and, second, the reduced model does reduce significantly the complexity in nonlinear dynamic analysis with large deformations.

As an example of this last statement, a model of a complete SensorCraft-like vehicle has been built by adding a flexible 1-m radius circular fuselage to the previous JW model. Its material properties are the same as for the wing skin. The resulting model represents the flexible vehicle including rigid-body motions (i.e., its flight dynamics degrees of freedom), and it can be used as a basic building block for nonlinear coupled flightdynamics and aeroelastic analysis of the complete aircraft. This provision is further justified because, for the undeformed vehicle, a fundamental first-bending linear vibration mode was identified at a frequency as low as $0.19 \mathrm{~Hz}$. In order to explore the behavior of this configuration, some nonlinear dynamic analyses were carried out under prescribed low-frequency loads for a vehicle clamped at the fuselage nose. Figure 18 shows the outer-wing tip displacements under a 2-Hz distributed harmonic load for $\mathrm{t}>0(1000 \mathrm{~N} / \mathrm{m}$ amplitude and 1000 $\mathrm{N} / \mathrm{m}$ mean value, which are values in the order of magnitude of the expected actual wing loadings) on all lifting surfaces. The excitation of the first linear vibration mode by the discontinuity at $\mathrm{t}=0$ in the average value of the loading (what can be considered as a linear effect) dominates the response, with wing tip deflections over $20 \%$ of wing semispan that bring significant geometrically nonlinear effects into play.
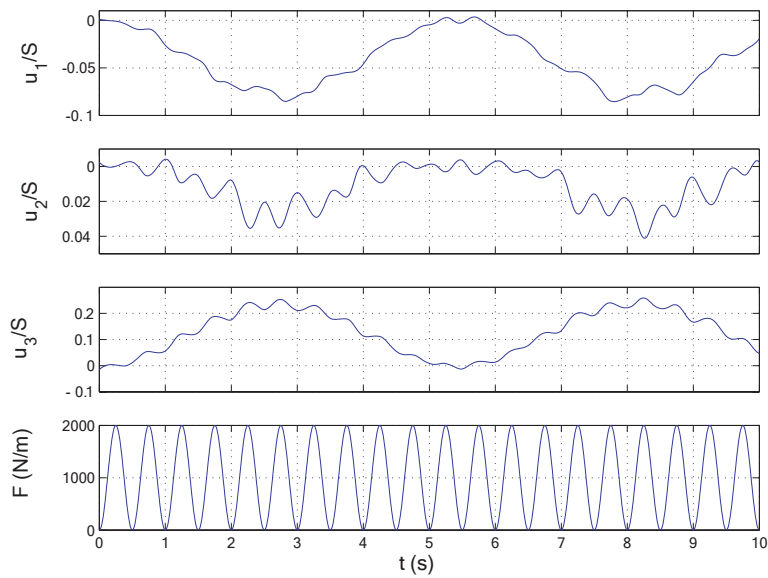

Figure 18. Tip displacements of the outer wing due to distributed harmonic loads normal to all lifting surfaces ( $S$ is the wing semispan)

Finally, the displacements of the original 3-D structure are also recovered at selected time steps in the simulation. As an example, Figure 19 shows the structure before deformation and in its deformed shape corresponding to maximum displacements at the wing tip. Figure 20 shows the corresponding 3-D dynamic stress distribution. These results complete the description of the dynamics of the original 3-D structure. Although in these analysis the representation of the deformation has used only the classical beam strains (extension, twist and bending), additional higher-order deformation modes (finite-section modes) could be easily included within the current reduced structural description. By doing so, the model could, for instance, be expanded to consider plate motions such as the camber bending of equation (7), or airfoils with an adaptive trailing edge, as in Ref. 21 .

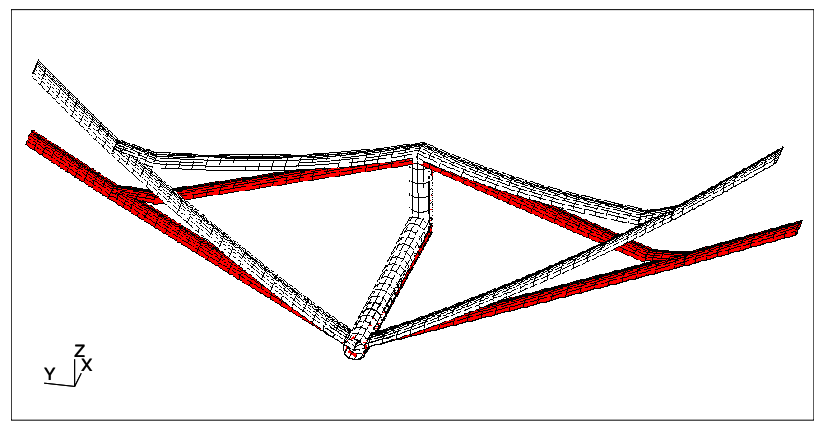

Figure 19. Undeformed and deformed vehicle at point of maximum tip displacement in the nonlinear response to the $2 \mathrm{~Hz}$ distributed harmonic force 


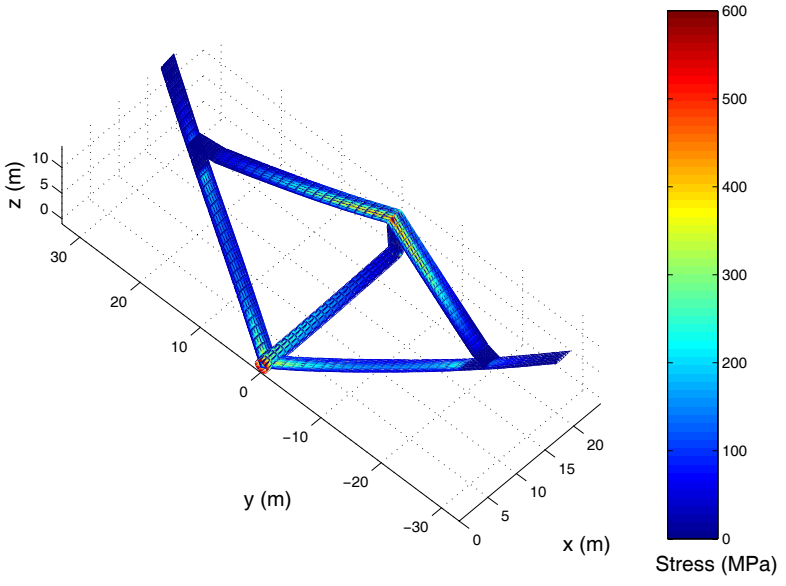

Figure 20. Von Mises dynamic stress field on the deformed structure at maximum tip displacement

\section{Conclusions}

A three-step modeling scheme for the construction of reduced models of active slender structures has been presented. It is shown how the 3-D electroelastic problem can be effectively solved in two independent problems. Higher-order deformations, as expected, for instance, in variable airfoil configurations or in structures with embedded actuation, are considered. The particular case of camber bending deformations induced by embedded actuators was studied for two configurations, and shown to compare well with plate/shell models. The methodology has been conceived for its future use in nonlinear aeroelastic analysis of complex, even morphing, configurations, and special attention has been given to its ability to represent 3-D displacement fields. This has been illustrated in the linear static response of a joined-wing configuration, including comparisons with an MSC.Nastran built-up model. A complete aircraft model was then built and its nonlinear response exemplified in an analysis with prescribed distributed loads. These results have shown the capability of the present approach to handle complex structural configurations within a low-order formulation.

\section{Acknowledgements}

The authors would like to sincerely thank Dr. Maxwell Blair (Air Force Research Laboratories) and Dr. Robert A. Canfield, USAF Lt. Col. (Air Force Institute of Technology) for providing the MSC.Nastran finiteelement models for the joined-wing aircraft used in this study. This work has been sponsored in part by the Army Research Office (grant 43854-EG) and NASA Langley Research Center (grant NAG-1-03038). The technical monitors are Dr. Gary L. Anderson and Dr. David M. Schuster, respectively.

\section{References}

1. Livne E, Weisshaar TA (2003). Aeroelasticity of nonconventional airplane configurations - Past and future. Journal of Aircraft, 40 (6) p 1047-1065

2. Rand O (2000). On the importance of cross-sectional warping in solid composite beams. Composite Structures 49 (4), p 393-397

3. Volovoi VV, Hodges DH, Cesnik CES, Popescu B (2001). Assessment of beam modeling methods for rotor blade applications. Mathematical and Computer Modelling 33 (1011), p 1099-1112

4. Jung SN, Nagaraj VT, Chopra I (1999). Assessment of composite rotor blade modeling techniques. Journal of the American Helicopter Society 44 (3), p 188-205

5. Berdichevsky VL (1982). On the energy of an elastic rod. PMM 45, p 518-529

6. Cesnik CES, Hodges DH (1997). VABS: A New Concept for Composite Rotor Blade Cross-Sectional Modeling. Journal of the American Helicopter Society 42 (1), p 27-38

7. Cesnik CES, Palacios R (2003). Modeling Piezocomposite Actuators Embedded in Slender Structures. Proc. 44th AIAA/ASME/ASCE/AHS/ASC Structures, Structural Dynamics, and Materials Conference. April 2003, Norfolk, Virginia

8. Chandra R, Chopra I (1993). Structural modeling of composite beams with induced-strain actuators. AIAA Journal 31 (9). p 1692-1701

9. McCarthy TR, Chattopadhyay A (1997). Refined higherorder composite box beam theory. Composites Part B: Engineering 28 (5-6), p 523-534

10. Centolanza LR, Smith EC (1998). Refined structural modeling of thick-walled closed section composite beams. Proc. $39^{\text {th }}$ AIAA/ASME/ASCE/AHS/ASC Structures, Structural Dynamics, and Materials Conference. April 1999, Long Beach, California

11. Volovoi VV, Hodges DH, Berdichevsky VL, Sutyrin VG (1998). Dynamic dispersion curves for non-homogeneous, anisotropic beams with cross-sections of arbitrary geometry. Journal of Sound and Vibration 215 (5), p 1101-1120

12. Chandra R, Chopra I (1993). Structural modeling of composite beams with induced-strain actuators. AIAA Journal 31 (9), p 1692-1701

13. Volovoi VV, Hodges DH (2000). Theory of Anisotropic Thin-Walled Beams. Journal of Applied Mechanics 67 (3), p 453-459

14. Cesnik CES, Sutyrin VG, Hodges DH (1996). Refined Theory of Composite Beams: The Role of Short-Wavelength Extrapolation. International Journal of Solids Structures, 33 (10), p 1387-1408

15. Palacios R, Cesnik CES (2003). Structural Dynamics of Integrally Strained Slender Wings. Proc. International Forum of Aeroelasticity and Structural Dynamics. June 2003, Amsterdam, The Netherlands

16. Tiersten HF (1969). Linear piezoelectric plate vibrations. 
Plenum Press, New York

17. Danielson DA, Hodges DH (1987). Nonlinear Beam Kinematics by Decomposition of the Rotation Tensor. ASME Journal of Applied Mechanics 54 (2), p 258-262

18. Brown SA (1997). Displacement Extrapolation for CFD and CSM Aeroelastic Analysis. Proc. $38^{\text {th }}$ AIAA/ASME/ASCE/AHS/ ASC Structures, Structural Dynamics, and Materials Conference. Apr 1997, Kissimmee, Florida

19. Cesnik CES, Shin S, Wilbur ML (2001). Dynamic response of active twist rotor blades. Smart Materials and Structures $\mathbf{1 0}$ (1), p 62-76

20. Bent AA (2000). Active fiber composite material systems for structural control applications. Proceedings of the 1999 SPIE Smart Structures and Materials Conference. March 2000, Newport Beach, California. p 166-177

21. Tilmann CP, Flick PM, Martin CA, and Love MH (2003). High-Altitude Long Endurance Technologies for SensorCraft. RTO AVT-099 Symposium on Novel and Emerging Vehicle and Vehicle Technology Concepts, RTO Paper MP-104-P-26, April 2003, Brussels, Belgium.

22. Blair M, Canfield RA (2002). A Joined-Wing Structural Weight Modeling Study. Proc. 43 $3^{\text {th }}$ AIAA/ASME/ASCE/AHS/ ASC Structures, Structural Dynamics, and Materials Conference. April 2002, Denver, Colorado

23. Roberts RW, Canfield RA, Blair M (2003). Sensor-Craft Structural Optimization and Analytical Certification. Proc. $44^{\text {th }}$ AIAA/ASME/ASCE/AHS/ASC Structures, Structural Dynamics, and Materials Conference, April 2003, Norfolk, Virginia

24. Gallman JW, Kroo IM (1996). Structural optimization for joined-wing synthesis. Journal of Aircraft 33 (1), p 214-223

25. Cesnik CES, Brown EL (2002). Modeling of High Aspect Ratio Active Flexible Wings for Roll Control.. Proc. $43^{\text {rd }}$ AIAA/ASME/ASCE/AHS/ASC Structures, Structural Dynamics, and Materials Conference. April 2002, Denver, Colorado

26. Livne E (2002). Aeroelasticity of Joined-Wing Airplane Configura-tions: Past Work and Future Challenges - A Survey. Proc. $42^{\text {nd }}$ AIAA/ASME/ASCE/AHS/ASC Structures, Structural Dynamics, and Materials Conference, April 2002, Seattle, Washington

27. Weisshaar TA, Lee DH (2002). Aeroelastic Tailoring of Joined-Wing Configurations. Proc. $43^{\text {rd }}$ AIAA/ASME/ASCE/ AHS/ASC Structures, Structural, Dynamics and Materials Conference, April 2002, Denver, Colorado 